\title{
Different types of Internet use, depression, and social anxiety: The role of perceived friendship quality
}

\author{
Maarten H.W. Selfhout*, Susan J.T. Branje ${ }^{1}$, M. Delsing ${ }^{2}$, \\ Tom F.M. ter Bogt ${ }^{3}$, Wim H.J. Meeus ${ }^{4}$ \\ Research Centre Adolescent Development, Utrecht University, P.O. Box 80140, 3508 TC, heidelberglaan 2, \\ Utrecht, The Netherlands
}

\begin{abstract}
The current study examined the longitudinal associations of time spent on Internet activities for communication purposes (i.e., IM-ing) versus time spent on Internet activities for non-communication purposes (i.e., surfing) with depression and social anxiety, as well as the moderating role of perceived friendship quality in these associations. Questionnaire data were gathered from 307 Dutch middle adolescents (average age 15 years) on two waves with a one-year interval. For adolescents who perceive low friendship quality, Internet use for communication purposes predicted less depression, whereas Internet use for non-communication purposes predicted more depression and more social anxiety. These results support social compensation effects of IMing on depression and poor-get-poorer effects of surfing on depression and social anxiety, respectively. (C) 2008 The Association for Professionals in Services for Adolescents. Published by Elsevier Ltd. All rights reserved.
\end{abstract}

Keywords: Internet use; Depression; Social anxiety; Perceived friendship quality

* Corresponding author. Tel.: +31 6471429 94; fax: +31 302537731 .

E-mail addresses: M.Selfhout@uu.nl (M.H.W. Selfhout), s.branje@uu.nl (S.J.T. Branje), marcd@socsci.ru.nl (M. Delsing), t.f.m.terbogt@uu.nl (T.F.M. ter Bogt), w.meeus@uu.nl (W.H.J. Meeus).

${ }^{1}$ Tel.: +313025340 39; fax. +31302537731.

${ }^{2}$ Tel.: +313025340 23; fax. +31302537731.

${ }^{3}$ Tel.: +313025347 40; fax. +31302537731.

${ }^{4}$ Tel.: +313025348 90; fax. +31302537731. 
Growing concern over the recent exponential increase of Internet use among teenagers has spawned a surge of research into the possible adverse effects of Internet use on well-being, with a primary focus on internalizing problems (e.g., Caplan, 2003; Gross, 2004; Kraut et al., 1998; Weiser, 2001). Recent US (Gross, Juvonen, \& Gable, 2002), Canadian (Hampton \& Wellman, 2001), and Dutch (Sikkema, 2005) studies have shown that the vast majority of adolescents spends several hours daily online. Further, surveys show that adolescents consider the Internet a highly important medium in their everyday social life and use it to form and maintain social relationships (Subrahmanyam, Smahel, \& Greenfield, 2006; Valkenburg \& Peter, 2007b; Wolak, Mitchell, \& Finkelhor, 2003). One theoretical approach to this phenomenon is that Internet use robs individuals of their social activities and might intervene with the development and maintenance of social relationships (e.g., Amichai-Hamburger \& Ben-Artzi, 2003; Caplan, 2003; Nie \& Erbring, 2000; Weiser, 2001). In line with this reasoning, several cross-sectional survey studies have found that Internet use was associated with more depression for adolescents (Cooper, 2006; Sun et al., 2005) and adults (Amichai-Hamburger \& Ben-Artzi, 2003; Caplan, 2003; Nie \& Erbring, 2000; Weiser, 2001). In contrast, another theoretical approach is that Internet use expands, rather than displaces, possibilities to contact peers, and thus enhances feelings of self-esteem and well-being (e.g., Morgan \& Cotten, 2003; Valkenburg \& Peter, 2007b). In accordance with this, crosssectional survey studies have shown that college students' Internet use was directly (Morgan \& Cotten, 2003) and indirectly (LaRose, Eastin, \& Gregg, 2001a) related to less depression. Furthermore, studies have revealed that Internet use can lead to online relationship formation, and thereby to more social support (Nie \& Erbring, 2000; Wellman, Quan-Haase, Witte, \& Hampton, 2001; Wolak et al., 2003), which may subsequently lead to less internalizing problems. One longitudinal study (Kraut et al., 1998) found that, over a period of 8-12 months, both loneliness and depression increased with time spent online among adolescent and adult first-time Internet users. In a one-year follow-up study (Kraut et al., 2002), however, the observed negative effects of Internet use had disappeared. Thus, studies concerning cross-sectional and longitudinal associations between Internet use and adolescent well-being have provided inconsistent results.

\section{Internet use for communication purposes versus Internet use for non-communication purposes}

The two previously mentioned theoretical approaches might adhere to different types of Internet activities that may have differential effects on well-being. One type of activities is surfing, which can be described as visiting websites on the Internet for non-communication purposes. Surfing seems to be strongly associated with Internet Addiction Disorder (Lijuan, Xin, Mingzheng, \& Aihong, 2006; Yang \& Tung, 2006; Zhu \& Wu, 2004). Symptoms of this disorder include preoccupation with Internet, deception regarding time spent online, mood modification through Internet use, and experiencing environmental distress. Being addicted to the Internet seems to lead to a vicious negative cycle, in which individuals receive short-term gratification every time they go online, making them want to go online more to seek this gratification (Hall \& Parsons, 2001). However, because surfing does not seem to have any long-term rewards usable in real-life, it does not seem to increase long-term gratification (LaRose, Mastro, \& Eastin, 2001b) and therefore may lead to depressive and socially anxious feelings (Morgan \& Cotten, 2003). In short, surfing may increase depression and social anxiety. 
In contrast to surfing, Instant Messaging (IM-ing) consists of sending messages directly to others one has invited to the online conversation. Because of its dyadic, real-time, and private format, IM-ing has been seen as an excellent 'training ground' for adolescents in terms of their social skills (Morgan \& Cotten, 2003; Valkenburg \& Peter, 2007b). Adolescents seem to have a high need for developing social skills and social relationships (Kiesner, Kerr, \& Stattin, 2004; Steinberg, 1996) and IM-ing may be seen as a relatively safe medium in which especially adolescents can practice and improve their social skills. Some researchers have suggested that with the increased individualization and physical distance between individuals and their family and peers in industrialized countries, less opportunities for normal face-to-face interaction with peers arise and IM-ing provides an excellent bridge across distance (Wolak et al., 2003). Thus, IM-ing might be a medium by which adolescents to interact with others, and could therefore be associated with the development of less depression and social anxiety.

Consistent with these expectations, a cross-sectional survey study among freshmen college students showed that whereas increased time spent IM-ing was uniquely associated with less reported depression, increased time spent surfing was uniquely associated with more reported depression (Morgan \& Cotten, 2003). In contrast, a recent longitudinal study showed that IM-ing predicted more depression over a six month period among 12-15 year old adolescents (Van den Eijnden, Meerkerk, Vermulst, Spijkerman, \& Engels, 2008). Further, although it has been suggested that socially anxious adolescents use Internet activities aimed at communication as a relatively safe way to explore social relationships (Shepherd \& Edelmann, 2005; Valkenburg, Schouten, \& Peter, 2005), Internet users spending most time IM-ing were not more socially anxious than Internet users spending less time IM-ing (Campbell, Cumming, \& Hughes, 2006). Therefore, although it seems conceptually important to distinguish between IM-ing and surfing when examining their links to internalizing problems, empirical studies thus far show inconsistent results regarding depression and indicate that IM-ing is not associated with social anxiety.

\section{Social compensation or rich-get-richer effects?}

Theories on effects of Internet use stress the need to consider moderating factors, such as individual traits and the social context in which Internet use occurs (Kraut et al., 1998; McKenna, 1999; Valkenburg \& Peter, 2007c). Because individual traits, especially Introversion, and their role in Internet use have received attention in several other studies (Amichai-Hamburger, Wainapel, \& Fox, 2002; Kraut et al., 1998; Peter, Valkenburg, \& Schouten, 2005), we will focus on one important aspect of the social context in adolescence: the quality of friendships. Theoretically, friendship quality may moderate Internet use effects on internalizing problems because it might reflect how the Internet is used by certain individuals: it might provide the motivation for using the Internet in a specific way. Two hypotheses are relevant in this respect. The first hypothesis is the rich-get-richer hypothesis, which suggests that IM-ing may have beneficial effects for individuals with high quality friendships, because IM-ing presents the ideal medium to maintain these friendships: its dyadic, realtime character makes it possible to closely interact with best friends. Adolescents with high quality friendships may also use IM-ing to expand their social network in a relatively easy way, because they may have better social skills which can be used to connect to new friends online (Anderson, 2001). In short, higher friendship quality may enhance beneficial effects of IM-ing on well-being. 
In contrast, the social compensation hypothesis states that in particular adolescents with low quality friendships will benefit from IM-ing, because constraints that make them interact poorly in real-life face-to-face encounters with their friends are not present so much in an online environment (Campbell et al., 2006; Walther, 1996). That is, adolescents with poor friendships may find the new online environment an ideal place to explore their identity (Valkenburg et al., 2005), develop their social skills (Wolak et al., 2003), and interact with new peers (Peter et al., 2005), which may all enhance their feelings of self-worth and well-being. Thus, IM-ing for adolescents with low quality friendships may reduce internalizing problems.

One study examined the role of friendship quality, thereby testing the social compensation hypothesis and the rich-get-richer hypothesis. For 11-17 year old adolescents who perceived low closeness with friends, chatting with strangers was cross-sectionally associated with higher wellbeing (Valkenburg \& Peter, 2007b). Thus, cross-sectional evidence supports the social compensation hypothesis, and challenges the rich-get-richer hypothesis when examining the role of friendship quality as a moderator in effects of Internet use on internalizing problems.

\section{The present study}

The present study extends prior research on the associations of Internet use with internalizing problems by examining the unique longitudinal associations of Internet activities for both communication purposes (i.e., IM-ing) and non-communication purposes (i.e., surfing) with depression and social anxiety. Because particularly adolescents use the Internet very frequently and may therefore be susceptible to influences of Internet use (Cooper, 2006; Gross et al., 2002; Valkenburg et al., 2005), we focus on the longitudinal effects of Internet use on internalizing problems in an adolescent sample. In addition, the moderating role of perceived friendship quality in these associations was examined to test two competing hypotheses derived from the rich-getricher perspective and the social compensation perspective. If IM-ing predicts fewer subsequent internalizing problems only for adolescents who perceive high friendship quality, the rich-getricher hypothesis would be supported. If, on the other hand, IM-ing predicts fewer subsequent internalizing problems only for adolescents who perceive low friendship quality, the social compensation hypothesis would be supported. We will explore whether time spent surfing predicts depression and social anxiety differentially for adolescents who perceive their friendship quality to be low and for adolescents who perceive their friendship quality to be high. Because prior researchers have suggested that girls may be more susceptible to adverse effects of IM-ing on wellbeing because they place relatively higher importance on the social aspects of Internet to communicate with best friends (Subrahmanyam et al., 2006), we will explore whether effects of Internet use on depression and social anxiety differ between boys and girls.

\section{Method}

\section{Participants}

Participants came from the early adolescent cohort participating in the CONflict And Management Of RElationships study (CONAMORE) (Meeus et al., 2004). Adolescents came 
from twelve high schools that were randomly selected in the province of Utrecht, the Netherlands. Questionnaires used in the present study were partially gathered at school (i.e., perceived friendship quality, depression, social anxiety) and partially at home (i.e., Internet use). For more details regarding the selection of the school and family sample of CONAMORE, see Van Doorn, Branje, and Meeus (2007). Questionnaires concerning Internet use were only filled out by individuals in the family sample $(n=307)$ at Wave 4 . We compared the 307 participants to the 323 non-participants on depression, social anxiety, and perceived friendship at Wave 4 and Wave 5. No significant differences ( $F$-values ranging from 0.19 to $0.87, p>0.10$ ) were found between these groups at either wave. From now on we will refer to Wave 4 as Time 1 and to Wave 5 as Time 2.

Of the 307 participants, 150 were boys $(48.8 \%)$ ). At Time 1 , the mean age of the adolescents was 15.5 years (ranging from 14 to 17 years, $\mathrm{SD}=0.6$ ). Most adolescents $(99.3 \%$ ) named Dutch as their main ethnic identity. Adolescents were relatively highly educated with approximately $51 \%$ of the adolescents at schools preparing for university, $34 \%$ of the adolescents at schools preparing for higher professional education, and $15 \%$ of the adolescents at schools preparing for blue-collar work.

\section{Procedure}

Before the study, both adolescents and their parents received written information and, if the adolescent wished to participate, were required to provide written informed consent. Interviewers visited the schools and asked participating adolescents to gather in classrooms to fill out a questionnaire. Interviewers also visited the families at home. During these home visits, adolescents filled out an additional questionnaire. Results were processed anonymously. Each wave, families received $€ 27$, for participating and adolescents received an additional amount of $€ 10$, - for participating at school.

\section{Measures}

\section{Depression}

Depression was assessed with the Children's Depression Inventory (CDI; Kovacs, 1992). This questionnaire contains 27 items. Example items include: "I feel sad all the time", "I often think other people do not like me", and "I hate myself". The items were scored on a 3-point scale, ranging from false, through a bit true, to true. A summed, total score was computed by adding the responses of all items. The CDI is a well-established instrument for measuring depression in nonclinical samples of children and adolescents (Craighead, Smucker, Craighead, \& Ilardi, 1998; Kovacs, 1992). The recommended clinical cut-off score used in community samples is 19 (Craighead, Curry, \& Ilardi, 1995; Craighead et al., 1998). In the current sample, this means that $4.8 \%(n=15)$ of the total sample score above the clinical cut-off score, which is comparable to what prior studies in the United States have found (Craighead et al., 1998). Furthermore, the mean score on depression for this specific sample $(M=5.19)$ was considerably lower than those reported for clinical samples $(M=12.56-16.25)$ (Comer \& Kendall, 2005; Craighead et al., 1998). The internal consistencies of this measure were 0.93 and 0.89 at Time 1 and 2, respectively.

\section{Social anxiety}

Social anxiety was measured by the Social Anxiety subscale of the revised version of the Screen for Child Anxiety Related Emotional Disorders (SCARED; Hale, Raaijmakers, Muris, 
$\&$ Meeus, 2005). The items were scored on a 3-point scale, ranging from almost never, through sometimes, to often. This scale contained 4 items: "I do not like to be around people I do not know", "I get nervous around people I do not know well", "I find it hard to talk to people I do not know", and "I get shy around people I do not know well". A total score was obtained by summing the responses to each item. Although no clinical cut-off scores are available for this measure, prior research among early to middle adolescents showed that this subscale of the SCARED is a valid measure of social anxiety in both non-clinical samples (Hale et al., 2005) and clinical samples (Birmaher et al., 1999). Furthermore, it has been demonstrated that the SCARED has strong sensitivity and specificity when compared with the Anxiety Disorders Interview Schedule for Children (A-DISC) in a study of schoolchildren (Muris, Merckelbach, Korver, \& Meesters, 2000). The mean score and variances on social anxiety for this specific sample $(n=307)$ did not differ significantly $(p>0.10)$ from means and variances of the total sample $(n=728)$. The internal consistencies of the social anxiety scale were 0.92 and 0.90 at Time 1 and 2, respectively.

\section{Internet use}

Adolescents were asked to fill out questions about Internet only if they ever used it. Of the 309 participating adolescents in the family sample, 307 (97.5\%) filled out questions about using Internet, namely surfing and IM-ing. Participants were first asked to make an estimation of the average weekly frequency spent chatting or surfing on a five-point scale, ranging from "never", through "less than once a week", through "about once a week", through "several times a week", to "every day". Next, participants estimated how much time on average they spent on an average weekly session of surfing and IM-ing on a four-point scale, ranging from shorter than $30 \mathrm{~min}$, through $30 \mathrm{~min}$ to $1 \mathrm{~h}$, through longer than $1 \mathrm{~h}$, to longer than $2 \mathrm{~h}$. A composite score surfing and IM-ing was calculated by multiplying the frequency they used IM-ing and surfing during the week by the time they spent IM-ing and surfing, respectively. Higher scores indicate that participants surf and IM more.

\section{Perceived friendship quality}

The Support scale of the Network of Relationship Inventory (NRI; Furman \& Buhrmester, 1985) was used to asses the target adolescent's perceived quality of the best friendship ${ }^{5}$. This scale contained 12 questions, such as: "How often do you turn to your best friend for support with personal problems?". Participants were asked to answer questions about relationship characteristics on a five-point scale $(1=$ never, $5=$ always $)$. The NRI has good predictive, factorial, and construct validity (Buhrmester \& Furman, 1987; Furman, 1996). Internal consistencies of support were 0.89 and 0.91 at Time 1 and 2 , respectively.

\footnotetext{
${ }^{5}$ The perception of friendship quality by the target adolescent may differ from the perception of friendship quality by the best friend. We checked whether the pattern of results was the same for models in which the perception of the target adolescent was replaced by perception of best friend. The pattern of associations turned out to be highly similar for both reporters: only for friends who perceived low friendship quality did IM-ing predict less depression $(\beta=-0.28$, $p<0.01)$. For the same group, surfing predicted more depression $(\beta=0.31, p<0.01)$ and social anxiety $(\beta=0.19$, $p<0.01)$. In the medium to high perceived friendship quality groups.
} 


\section{Statistical analysis}

To examine the effects of Internet use on depression and social anxiety, two series of hierarchical regression analyses were performed: one with depression at Time 2 as dependent variable, and one with social anxiety at Time 2 as dependent variable. For each step, we tested whether adding the additional variables resulted in a significant $(p<0.05)$ increase in explained variance. In step 1 , we entered sex $(0=$ male, $1=$ female $)$, depression, and social anxiety at Time 1 to predict depression and social anxiety at Time 2 . In step 2, perceived friendship quality, time spent IM-ing, and time spent surfing at Time 1 were used to predict depression and social anxiety at Time 2, thereby controlling for the previously entered variables. In step 3, we included four additional variables: the interaction between IM-ing and friendship quality at Time 1, the interaction between surfing and friendship quality at Time 1, the interaction between sex and IM-ing at Time 1, and the interaction between sex and surfing at Time 1. All independent variables were centred to avoid multicollinearity.

If in the last step one of the interaction terms was significant, we used post-hoc testing according to formulas of Aiken and West (1991) for the variables included in the significant interaction term in question. We trichotomized friendship quality as follows. Scores one standard deviation above and below the mean of perceived friendship support at Time 1 were used to make three perceived friendship quality groups. Those adolescents scoring one standard deviation below the mean at Time 1 were labeled low friendship quality group $(n=81)$ and those scoring one standard deviation above the mean at Time 1 were labeled high friendship quality group $(n=61)$. Other adolescents were labeled medium friendship quality group $(n=165)$. Finally, we explored differences between boys and girls in interaction effects between Internet use and perceived friendship quality by entering the 3-way interaction between perceived friendship quality, IM-ing, and sex, as well as the 3-way interaction between perceived friendship quality, surfing, and sex, while controlling for all previously mentioned independent variables.

\section{Results}

In Table 1, descriptive results of Internet use and internalizing problems for the total sample can be found. Additionally, we examined differences between the low, medium, and high perceived friendship quality groups, to provide more insight into the moderating role of friendship quality in the link between Internet use and internalizing problems. A MANOVA showed significant mean differences between the low, medium, and high best friendship quality groups regarding means of Internet use, depression at Time 1 and Time 2, and social anxiety at Time 1 and Time 2: $F(14,590)=5.28, p<0.01)$. Bonferoni post-hoc tests showed that adolescents with low perceived friendship quality spent significantly $(p<0.05)$ less time IM-ing than adolescents with high perceived friendship quality at Time 1 . In addition, adolescents with low perceived friendship quality reported significantly $(p<0.01)$ higher depression and social anxiety at both Time 1 and Time 2 compared to adolescents with medium to high friendship quality. 
Table 1

Descriptive statistics of Internet use and internalizing problems by low, medium, and high perceived friendship quality groups.

\begin{tabular}{|c|c|c|c|c|}
\hline \multirow[t]{3}{*}{ Dimension } & \multicolumn{4}{|l|}{ Mean $(S D)$} \\
\hline & All & Low & Medium & High \\
\hline & $(n=307)$ & $(n=81)$ & $(n=165)$ & $(n=61)$ \\
\hline $\begin{array}{l}\mathrm{T} 1 \text { perceived } \\
\text { friendship quality }\end{array}$ & $3.87(0.92)$ & $2.89(0.42)$ & $3.85(0.38)$ & $4.86(0.20)$ \\
\hline $\begin{array}{l}\mathrm{T} 2 \text { perceived } \\
\text { friendship quality }\end{array}$ & $3.86(0.74)$ & $2.91(0.38)$ & $3.85(0.31)$ & $4.83(0.23)$ \\
\hline T1 IM-ing & $8.24(4.83)$ & $7.42(4.53)^{\mathrm{a}}$ & $8.01(4.94)^{\mathrm{ab}}$ & $9.23(5.02)^{\mathrm{b}}$ \\
\hline T1 surfing & 7.85 (4.28) & $8.07(4.22)^{\mathrm{a}}$ & $7.69(4.11)^{\mathrm{a}}$ & $7.64(4.41)^{\mathrm{a}}$ \\
\hline $\mathrm{T} 1$ depression & $1.23(0.22)$ & $1.32(0.22)^{\mathrm{a}}$ & $1.21(0.16)^{\mathrm{b}}$ & $1.18(0.25)^{\mathrm{b}}$ \\
\hline $\mathrm{T} 1$ anxiety & $1.24(0.25)$ & $1.29(0.23)^{\mathrm{a}}$ & $1.21(0.22)^{\mathrm{b}}$ & $1.20(0.28)^{\mathrm{b}}$ \\
\hline T2 depression & $1.25(0.19)$ & $1.30(0.20)^{\mathrm{a}}$ & $1.22(0.15)^{\mathrm{b}}$ & $1.17(0.21)^{\mathrm{b}}$ \\
\hline $\mathrm{T} 2$ anxiety & $1.23(0.23)$ & $1.27(0.22)^{\mathrm{a}}$ & $1.23(0.24)^{\mathrm{b}}$ & $1.20(0.25)^{\mathrm{b}}$ \\
\hline
\end{tabular}

Note. Numbers in each cell refer to means and standard deviations (between brackets), respectively. Differing superscripts $\left({ }^{\mathrm{a}, \mathrm{b}}\right)$ indicate significant $(p<0.05)$ differences between low, medium, and high quality groups.

Longitudinal associations between internet use, friendship quality, depression, and social anxiety over time

Table 2 shows the results of the hierarchical regression analyses concerning the longitudinal associations of Internet use and perceived friendship quality with depression and social anxiety at Time 2. In step 1, sex, depression, and social anxiety at Time 1 were used to predict depression and social anxiety at Time 2 . Results showed that variance explained by these variables was significant: $F(3,303)=46.09, p<0.05$, for depression, and $F(3,303)=57.22, p<0.05$, for social anxiety. First, girls tended to be more depressed and more socially anxious than boys. Further, medium rank-order stability of depression and social anxiety was found across a one-year period.

In step 2, we examined main effects of Internet use as well as perceived friendship quality on depression and social anxiety over time. Therefore, IM-ing, surfing, and friendship quality at Time 1 were additionally included in the regression analyses. Results showed that variance explained by these variables did not significantly increase for depression $(F(6,300)=1.09$, $p>0.05)$ and for social anxiety $(F(6,300)=0.22, p>0.05)$. Specifically, IM-ing, surfing, and perceived friendship quality at Time 1 did not predict depression or social anxiety at Time 2. Thus, for the sample as a whole, both IM-ing and surfing are not longitudinally associated with depression and social anxiety over time.

Next, we tested whether perceived friendship quality moderated longitudinal associations between Internet use and internalizing problems. Thus, at step 3, we included the interactions of friendship quality with IM-ing and surfing at Time 1, while controlling for all previous effects. Interactions of sex with IM-ing and surfing were also included. Results showed that variance explained by these variables increased significantly for depression $(F(10,296)=25.09, p<0.05)$ and for social anxiety $(F(10,296)=10.22, p<0.05)$. Specifically, whereas the interaction between IM-ing and perceived friendship quality predicted less depression at Time 2, the interaction between surfing and perceived friendship quality at Time 1 predicted more depression at Time 2 . 
Table 2

Longitudinal associations between depression, social anxiety, Internet use and friendship quality at Time 1 and depression and social Anxiety at Time 2.

\begin{tabular}{|c|c|c|}
\hline & Depression T2 & Social anxiety $\mathrm{T} 2$ \\
\hline \multicolumn{3}{|l|}{ Step 1} \\
\hline Sex $(1=$ female $)$ & $0.15^{* *}$ & $0.08 *$ \\
\hline Social anxiety $\mathrm{T} 1$ & $0.10^{*}$ & $0.61 * *$ \\
\hline Depression T1 & $0.50 * *$ & 0.02 \\
\hline$R^{2}$ change & $0.29 * *$ & $0.38 * *$ \\
\hline \multicolumn{3}{|l|}{ Step 2} \\
\hline Sex $(1=$ female $)$ & -0.05 & -0.06 \\
\hline Social anxiety $\mathrm{T} 1$ & $0.09 *$ & $0.59 * *$ \\
\hline Depression T1 & $0.49 * *$ & 0.02 \\
\hline IM-ing T1 & -0.03 & -0.01 \\
\hline Surfing T1 & 0.06 & 0.02 \\
\hline Friendship quality $\mathrm{T} 1$ & -0.03 & -0.08 \\
\hline$R^{2}$ change & 0.01 & 0.01 \\
\hline \multicolumn{3}{|l|}{ Step 3} \\
\hline Sex $(1=$ female $)$ & -0.05 & -0.06 \\
\hline Social anxiety $\mathrm{T} 1$ & $0.08^{*}$ & $0.59 * *$ \\
\hline Depression T1 & $0.49 * *$ & 0.02 \\
\hline IM-ing T1 & -0.02 & -0.00 \\
\hline Surfing T1 & 0.06 & 0.02 \\
\hline Friendship quality $\mathrm{T} 1$ & -0.02 & -0.05 \\
\hline IM-ing $\mathrm{T} 1 \times$ friendship quality $\mathrm{T} 1$ & $-0.23 * *$ & 0.03 \\
\hline Surfing $\mathrm{T} 1 \times$ friendship quality $\mathrm{T} 1$ & $0.20 * *$ & $0.13^{*}$ \\
\hline Sex $\times$ IM-ing T1 & 0.00 & 0.00 \\
\hline Sex $\times$ surfing $\mathrm{T} 1$ & 0.00 & 0.01 \\
\hline$R^{2}$ change & $0.09 * *$ & $0.02 *$ \\
\hline Total $R^{2}$ & 0.38 & 0.40 \\
\hline
\end{tabular}

Note. All independent variables were centered. T1 $=$ Time $1 ; \mathrm{T} 2=$ Time 2.

$* p<0.05 ; * * p<0.01$.

Further, the interaction between surfing and perceived friendship quality at Time 1 predicted more social anxiety at Time 2. Interaction effects of sex with IM-ing and surfing at Time 1 on depression and social anxiety at Time 2 were not significant, indicating that effects of IM-ing and surfing on depression and social anxiety did not differ between boys and girls.

We used post-hoc testing according to Aiken and West's (1991) formulas to follow-up the significant interactions, assessing the effect of IM-ing and surfing for groups of adolescents with low, medium and high friendship quality. First, higher levels of IM-ing at Time 1 were associated with less depression at Time 2 only for adolescents with low friendship quality (see Fig. 1: $\beta=-0.27, p<0.01 ; \beta=0.03, p>0.05 ; \beta=0.02, p>0.05$, for low, medium, and high friendship quality, respectively). Second, higher levels of surfing at Time 1 were associated with more depression at Time 2 only for adolescents with low friendship quality (see Fig. 2: $\beta=0.33$, $p<0.01 ; \beta=0.04, p>0.05 ; \beta=0.05, p>0.05$, for low, medium, and high friendship quality, respectively). Third, higher levels of surfing at Time 1 were associated with more social anxiety at 


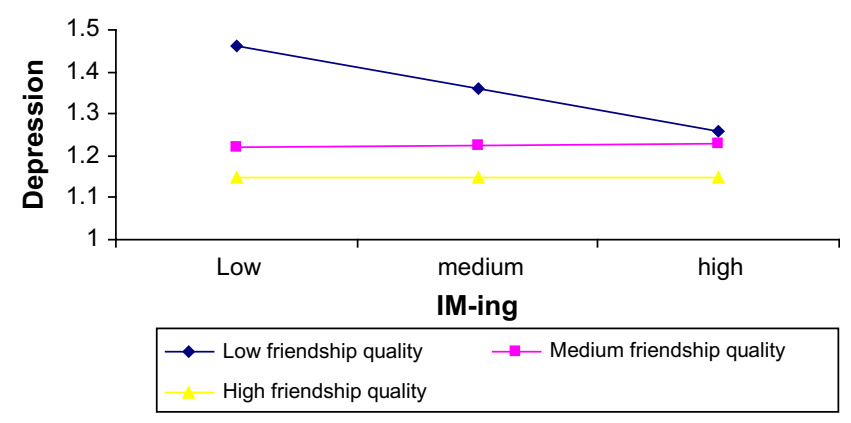

Fig. 1. Associations between IM-ing at Time 1 and depression at Time 2 for adolescents with low, medium, and high perceived friendship quality.

Time 2 only for adolescents with low friendship quality (see Fig. $3: \beta=0.22, p<0.01 ; \beta=0.01$, $p>0.05 ; \beta=0.03, p>0.05$, for low, medium, and high friendship quality, respectively).

Finally, we explored three-way interactions between perceived friendship quality, IM-ing, and sex, and between perceived friendship quality, surfing, and sex to test whether boys and girls differ in interaction effects between internet use and perceived friendship quality. Because none of these three-way interaction terms $(F(2,303)=1.02, p>0.05$, for depression, and $(F(2,303)=0.33$, $p>0.05$ ), for social anxiety) added significantly to the model, we omitted these interaction terms from Table 2.

In sum, friendship quality seems to moderate longitudinal associations between time spent on the Internet and internalizing problems: only for adolescents with low perceived quality of friendship, time spent IM-ing predicted less depression, and time spent surfing predicted more depression and social anxiety over a one-year period. In the group that perceived medium to high friendship quality, no associations between time spent IM-ing or surfing and internalizing problems were found.

\section{Discussion}

The present study examined the longitudinal associations between time spent on Internet activities for communication purposes (i.e., IM-ing) and time spent on Internet activities for noncommunication purposes (i.e., surfing) with depression and social anxiety. Results showed no

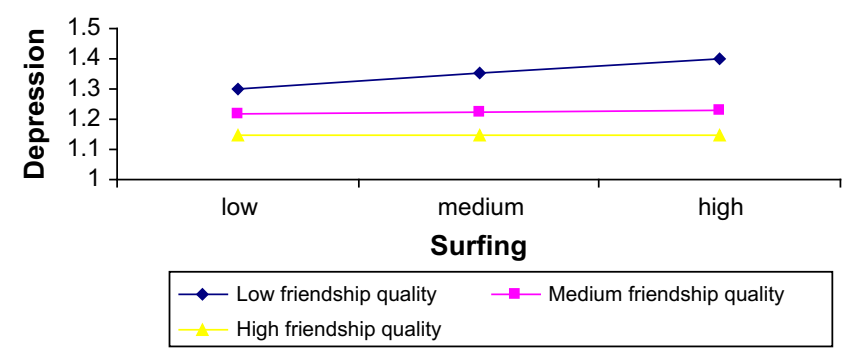

Fig. 2. Associations between surfing at Time 1 and depression at Time 2 for adolescents with low, medium, and high perceived friendship quality. 


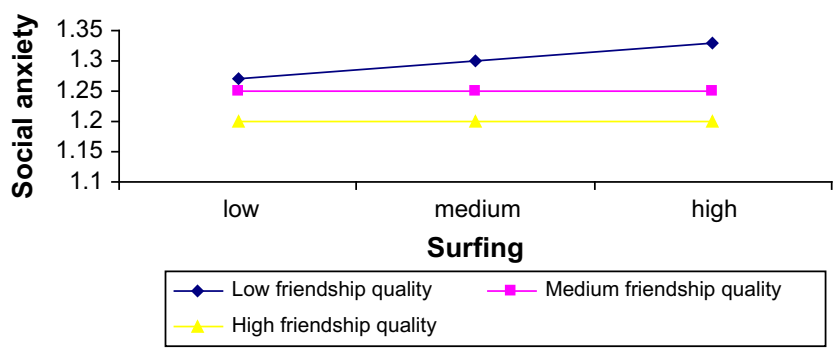

Fig. 3. Associations between surfing at Time 1 and social anxiety at Time 2 for adolescents with low, medium, and high perceived friendship quality.

longitudinal associations between either time spent IM-ing or time spent surfing and changes in internalizing problems for the sample as a whole. Although a previous study has found IM-ing to predict more depression over a six month period (Van den Eijnden et al., 2008), this study did not take the unique associations of IM-ing and surfing with depression into account. Effects of IM-ing on internalizing problems may be confounded with effects of surfing on internalizing problems, since adolescents who spend more time IM-ing also tend to spend more time surfing (Subrahmanyam, Greenfield, Kraut, \& Gross, 2001). The results of the current study suggest that IMing or surfing do not affect adolescent internalizing problems for adolescents in general. Thus, no support was found for generalized "bad versus good" effects of Internet use on youth, and the refore the context in which Internet use occurs needs to be taken into account.

As expected, we found that the social context in which Internet use occurs moderates effects of Internet use on internalizing problems, perhaps because the social context may pertain to how the Internet is used (Kraut et al., 1998; McKenna, 1999; Valkenburg \& Peter, 2007c; Whitlock, Powers, \& Eckenrode, 2006). For adolescents with low quality friendships, surfing longitudinally predicted more depression and more social anxiety. Time spent surfing may have adverse effects on well-being only for these adolescents because especially for these adolescents, surfing might be linked with Internet Addiction Disorder (Yang \& Tung, 2006). Surfing may be linked to a negative cycle in which going online creates direct gratification, but no long-term gratification (LaRose et al., 2001b). This result provides evidence for what could be called 'poor-get-poorer' effects: adolescents with already low quality friendships who spend more time on Internet activities for non-communication purposes may develop more internalizing problems.

In contrast, if adolescents with low quality friendships spend more time IM-ing, they seem to develop less depression over time. IM-ing may increase their chance of getting (or perceiving) social support and may strengthen their social skills, because these adolescents can safely explore peer relationships from behind their computer (Morgan \& Cotten, 2003; Peter et al., 2005; Subrahmanyam et al., 2006). However, no longitudinal social compensation effects were found for social anxiety: IM-ing did not predict less social anxiety over time for adolescents who perceive low friendship quality. Although for adolescents with low social skills, IM-ing might increase the feeling that one is supported and as such lead to less depression (Peter et al., 2005), results of the current study confirm suggestions that IM-ing does not reduce the fear of 'off-line' social situations (Campbell et al., 2006). Future research should examine whether increased social skills and perceived social support are indeed the mechanisms that explain the link between IM-ing and the development of less depression. 
Even though results showed that adolescents with high quality friendships use IM-ing even more than adolescents with low quality friendships, no support was found for rich-get-richer effects: surfing and IM-ing seem to have neither beneficial nor detrimental effects on internalizing problems of adolescents with high quality friendships. These adolescents may be less prone to adverse influences from Internet activities for non-communication purposes as they have high quality friendships to fall back upon. At the same time, they may not need Internet activities for communication purposes due to already strong social skills or strong ties to friends (Peter et al., 2005). Note that all the previously discussed results apply to both boys and girls, which is consistent with recent evidence showing gender differences in Internet use and effects of Internet use on well-being to be not as sizeable as they once were (Gross, 2004; Valkenburg et al., 2005).

Several limitations to this study should be noted. First, the sample consisted of relatively highly educated adolescents from two-parent families, and future studies need to examine whether results of the present study can be generalized to other samples. However, a study using a sample of adolescents with lower and higher educational backgrounds also showed that online communication was cross-sectionally associated with less social anxiety for adolescents who experienced low closeness to friends (Valkenburg \& Peter, 2007a), suggesting that the current results also apply to adolescents with lower educational backgrounds. Nevertheless, future studies should examine differences between adolescents with low versus high educational backgrounds and adolescents with different family structures regarding effects of Internet use on well-being. Second, the current study assessed the perception of friendship quality by the target adolescent, which may differ from perceptions of the best friends and from objective criteria concerning friendship quality. However, it may be especially the perception of friendship quality that is most consequential for Internet use and adolescent adjustment instead of the actual friendship quality. For example, whether friendships actually are of low quality may be of minor relevance to adolescent Internet use; the perception of friendships may guide adolescents' actions more than the actual state of these friendships. In addition, if self-report data on perceived friendship quality are biased in some way, this bias would be present in all three friendship quality groups and can therefore not explain differences across groups in effects of Internet use on depression and social anxiety. Additional analyses using the perception of the best friend instead of the target adolescent showed similar results, which suggests that these effects are independent of the source of information on friendship quality. A third limitation of the present study is that longitudinal data were available only on depression and social anxiety, not on Internet use. Results only provide evidence for an association between earlier Internet use and later well-being. Although the longitudinal design of the present study allows for conclusions about predictive effects of Internet use on subsequent internalizing problems, strong claims on causality should be made with caution: results cannot definitively prove whether Internet use leads to less or more internalizing problems. The causal chain may be different: individuals who feel more depressed and social anxious may start surfing more and IM-ing less. Future studies should examine the bidirectional longitudinal associations between different types of Internet use and internalizing problems.

In sum, the present study suggests that Internet use may have beneficial as well as detrimental effects on adolescent internalizing problems. Whereas Internet use based on communication seems to have beneficial effects on depression for adolescents who report having poor friendships, Internet use based on non-communication seems to have detrimental effects on these adolescents' depression and social anxiety. Adolescents who report having medium to high quality friendships 
seem unaffected by either type of Internet use. Thus, models regarding Internet use are needed that stress effects of Internet use on adolescent internalizing problems depending on both the type of Internet use and the social context in which Internet use occurs.

\section{References}

Aiken, L. S., \& West, S. G. (1991). Multiple regression: Testing and interpreting interactions. Newbury Park, CA: Sage Publications.

Amichai-Hamburger, Y., \& Ben-Artzi, E. (2003). Loneliness and Internet use. Computers in Human Behavior, 19, 71-80.

Amichai-Hamburger, Y., Wainapel, G., \& Fox, S. (2002). On the Internet no one knows I'm an introvert': extroversion, neuroticism, and Internet interaction. CyberPsychology and Behavior, 5, 125-128.

Anderson, K. J. (2001). Internet use among college students: an exploratory study. Journal of American College Health, $50,21-26$.

Birmaher, B., Brent, D. A., Chiappetta, L., Bridge, J., Monga, S., \& Baugher, M. (1999). Psychometric properties of the Screen for Child Anxiety Related Emotional Disorders (SCARED): A replication study. Journal of the American Academy of Child and Adolescent Psychiatry, 38, 1230-1236.

Buhrmester, D., \& Furman, W. (1987). The development of companionship and intimacy. Child Development, 58, 1101-1113.

Campbell, A. J., Cumming, S. R., \& Hughes, I. (2006). Internet use by the socially fearful: addiction or therapy? CyberPsychology and Behavior, 9, 69-81.

Caplan, S. E. (2003). Preference for online social interaction: a theory of problematic Internet use and psychosocial well-being. Communication Research, 30, 625-648.

Comer, J. S., \& Kendall, P. C. (2005). High-end specifity of the Children's Depression Inventroy in a sample of anxietydisordered. Depression and Anxiety, 22, 11-19.

Cooper, N. S. (2006). The identification of psychological and social correlates of Internet use in children and teenagers. Dissertation Abstracts International: Section B: The Sciences and Engineering, 66.

Craighead, W. E., Curry, J. F., \& Ilardi, S. S. (1995). Relationship of Children's Depression Inventory factors to major depression among adolescents. Psychological Assessment, 7, 171-176.

Craighead, W. E., Smucker, M. R., Craighead, L. W., \& Ilardi, S. S. (1998). Factor analysis of the Children's Depression Inventory in a community sample. Psychological Assessment, 10, 156-165.

Furman, W. (1996). The measurement of friendship perceptions: conceptual and methodological issues. In W. M. Bukowski, A. F. Newcomb, \& W. W. Hartup (Eds.), The company they keep: Friendship in childhood and adolescence (pp. 41-65). New York, NY: Cambridge University Press.

Furman, W., \& Buhrmester, D. (1985). Children's perceptions of the personal relationship in their social networks. Developmental Psychology, 21, 1016-1024.

Gross, E. F. (2004). Adolescent Internet use: what we expect, what teens report. Journal of Applied Developmental Psychology, 25, 633-649.

Gross, E. F., Juvonen, J., \& Gable, S. L. (2002). Internet use and well-being in adolescence. Journal of Social Issues, 58, 75-90.

Hale, W. W., III, Raaijmakers, Q., Muris, P., \& Meeus, W. (2005). Psychometric properties of the Screen for Child Anxiety Related Emotional Disorders (SCARED) in the general adolescent population. Journal of the American Academy of Child and Adolescent Psychiatry, 44, 283-290.

Hall, A. S., \& Parsons, J. (2001). Internet addiction: college student case study using best practices in cognitive behavior therapy. Journal of Mental Health Counseling, 23, 312-327.

Hampton, K., \& Wellman, B. (2001). Long distance community in the network society. American Behavioral Science, 45, 476-495.

Kiesner, J., Kerr, M., \& Stattin, H. (2004). Very important persons” in adolescence: going beyond in-school, single friendships in the study of peer homophily. Journal of Adolescence, 27, 545-560.

Kovacs, M. (1992). Children's Depression Inventory, CDI Manual. North Tonawanda, NY: Multi-Health Systems. 
Kraut, R., Kiesler, S., Boneva, B., Cummings, J. N., Helgeson, V., \& Crawford, A. M. (2002). Internet paradox revisited. Journal of Social Issues, 58, 49-74.

Kraut, R., Patterson, M., Lundmark, V., Kiesler, S., Mukophadhyay, T., \& Scherlis, W. (1998). Internet paradox: a social technology that reduces social involvement and psychological well-being? American Psychologist, 53, 1017-1031.

LaRose, R., Eastin, M. S., \& Gregg, J. (2001a). Reformulating the Internet paradox: social cognitive explanations of Internet use and depression. Journal of Online Behavior, 1, 1-19.

LaRose, R., Mastro, D., \& Eastin, M. S. (2001b). Understanding Internet usage: a social-cognitive approach to uses and gratifications. Social Science Computer Review, 19, 395-413.

Lijuan, C., Xin, Z., Mingzheng, W., \& Aihong, X. (2006). A research on the effects of Internet addiction on adolescents' social development. Psychological Science (China), 29, 34-36.

McKenna, K. Y. A. (1999). The computers that bind: relationship formation on the Internet. Dissertation Abstracts International Section A: Humanities and Social Sciences, 59.

Meeus, W., Akse, J., Branje, S., Ter Bogt, T., Engels, R., Finkenauer, C., et al. (2004). Codebook CONAMORE: conflicts and management of RELATIONSHIPS. Utrecht University, Faculty of Social Sciences, unpublished manuscript.

Morgan, C., \& Cotten, S. R. (2003). The relationship between Internet activities and depressive symptoms in a sample of college freshmen. CyberPsychology and Behavior, 6, 133-142.

Muris, P., Merckelbach, H., Korver, P., \& Meesters, C. (2000). Screening for trauma in children and adolescents: the validity of the traumatic stress disorder scale of the screen for child anxiety related Emotional disorders. Journal of Clinical Child Psychology, 29, 406-413.

Nie, N. H., \& Erbring, L. (2000). Internet and society: A preliminary report. Stanford, CA: Stanford Inst. of Quant. Study Soc.

Peter, J., Valkenburg, P. M., \& Schouten, A. P. (2005). Developing a model of adolescent friendship formation on the Internet. CyberPsychology and Behavior, 8, 423-430.

Shepherd, R.-M., \& Edelmann, R. J. (2005). Reasons for Internet use and social anxiety. Personality and Individual Differences, 39, 949-958.

Sikkema, P. (2005). Jongeren 2005. [Youngsters 2005]. Amsterdam: Audax Publishing/Media B.V.

Steinberg, L. (1996). Adolescence. New York: McGraw-Hill.

Subrahmanyam, K., Greenfield, P., Kraut, R., \& Gross, E. (2001). The impact of computer use on children's and adolescents' development. Journal of Applied Developmental Psychology, 22, 7-30.

Subrahmanyam, K., Smahel, D., \& Greenfield, P. (2006). Connecting developmental constructions to the Internet: identity presentation and sexual exploration in online teen chat rooms. Developmental Psychology, 42, 395-406.

Sun, P., Unger, J. B., Palmer, P. H., Gallaher, P., Chou, C.-P., Baezconde-Garbanati, L., et al. (2005). Internet accessibility and usage among urban adolescents in southern California: implications for web-based health research. CyberPsychology and Behavior, 8, 441-453.

Valkenburg, P. M., \& Peter, J. (2007a). Internet communication and its relationship to wellbeing: identifying some underlying mechanisms. Media Psychology, 9, 43-58.

Valkenburg, P. M., \& Peter, J. (2007b). Online communication and adolescent well-being: testing the stimulation versus the displacement hypothesis. Journal of Computer Mediated Communication, 12, 1169-1182.

Valkenburg, P. M., \& Peter, J. (2007c). Preadolescents' and adolescents' online communication and their closeness to friends. Developmental Psychology, 43, 267-277.

Valkenburg, P. M., Schouten, A. P., \& Peter, J. (2005). Adolescents' identity experiments on the Internet. New Media and Society, 7, 383-402.

Van den Eijnden, R. J. J. M., Meerkerk, G.-J., Vermulst, A. A., Spijkerman, R., \& Engels, R. C. M. E. (2008). Online communication, compulsive Internet use and psychosocial well-being among adolescents: a longitudinal study. Developmental Psychology, 44, 655-665.

Van Doorn, M. D., Branje, S., \& Meeus, W. (2007). Longitudinal transmission of conflict resolution styles from marital relationships to adolescent-parent relationships. Journal of Family Psychology, 21, 426-434.

Walther, J.-B. (1996). Computer-mediated communication: impersonal, interpersonal, and hyperpersonal interaction. Communication-Research, 23(1), 3-43.

Weiser, E. B. (2001). The functions of Internet use and their social and psychological consequences. CyberPsychology and Behavior, 4, 723-743. 
Wellman, B., Quan-Haase, A., Witte, J., \& Hampton, K. (2001). Does the Internet increase, decrease, or supplement social capital? Social networks, participation, and community commitment. American Behavioral Scientist, 45, 436-455.

Whitlock, J. L., Powers, J. L., \& Eckenrode, J. E. (2006). The virtual cutting edge: the Internet and adolescent selfInjury. Developmental Psychology, 240, 407-417.

Wolak, J., Mitchell, K. J., \& Finkelhor, D. (2003). Escaping or connecting? Characteristics of youth who form close online relationships. Journal of Adolescence, 26, 105-119.

Yang, S. C., \& Tung, C.-J. (2006). Comparison of Internet addicts and non-addicts in Taiwanese high school. Computers in Human Behavior, 23, 79-96.

Zhu, K., \& Wu, H. (2004). Psychosocial factors of to Internet addiction disorder in college students. Chinese Mental Health Journal, 18, 796-798. 